www.nature.com $/ \mathrm{hr}$

\title{
Refined mapping of blood pressure quantitative trait loci using congenic strains developed from two genetically hypertensive rat models
}

\author{
Sivarajan Kumarasamy ${ }^{1}$, Kathirvel Gopalakrishnan ${ }^{1}$, Edward J Toland ${ }^{1}$, Shane Yerga-Woolwine ${ }^{1}$, \\ Phyllis Farms ${ }^{1}$, Eric E Morgan ${ }^{2}$ and Bina Joe ${ }^{1}$
}

Previously linkage and substitution mapping were conducted between the Dahl Salt-sensitive (S) rat and the Spontaneously Hypertensive Rat (SHR) to address the hypothesis that genetic contributions to blood pressure (BP) in two genetically hypertensive rat strains are different. Among the BP quantitative trait loci (QTLs) detected, two are located on chromosome 9 within large genomic segments. The goal of the current study was to develop new iterations of congenic substrains, to further resolve both of these BP QTLs on chromosome 9 as independent congenic segments. A total of 10 new congenic substrains were developed and characterized. The newly developed congenic substrains S.SHR(9)x8Ax11A and S.SHR(9)x10Ax1, with introgressed segments of 2.05 and $6.14 \mathrm{Mb}$, represented the shortest genomic segments. Both of these congenic substrains, S.SHR(9)x8Ax11A and S.SHR(9)x10Ax1 lowered BP of the $S$ rat by $56 \mathrm{~mm} \mathrm{Hg}(P<0.001)$ and $15 \mathrm{~mm} \mathrm{Hg}(P<0.039)$, respectively. The BP measurements were corroborated by radiotelemetry. Urinary protein excretion was significantly lowered by SHR alleles within S.SHR(9)x10Ax1 but not by S.SHR(9)x8Ax11A. The shorter of the two congenic segments, $2.05 \mathrm{Mb}$ was further characterized and found to contain a single differentially expressed protein-coding gene, Tomoregulin-2 (Tmeff2). The protein expression of Tmeff2 was higher in the S rat compared with S.SHR(9)x8Ax11A, which also had lower cardiac hypertrophy as measured by echocardiography. Tmeff2 is known to be upregulated in patients from multiple cohorts with cardiac hypertrophy. Taken together, Tmeff 2 can be prioritized as a candidate gene for hypertension and associated cardiac hypertrophy in both rats and in humans.

Hypertension Research (2011) 34, 1263-1270; doi:10.1038/hr.2011.116; published online 4 August 2011

Keywords: Dahl S rat; SHR; Tomoregulin-2

\section{INTRODUCTION}

Blood Pressure (BP) is a complex polygenic trait well studied in both model organisms and in humans. Genome-wide association studies in humans have identified a few regions and candidate genes underlying BP regulation. ${ }^{1-11}$ However, the extent of genetic heritability accounted by association studies alone does not represent the total heritability. ${ }^{12}$ Therefore, identifying the key determinants of BP control remains to be a major challenge.

Classic linkage analyses and substitution mapping studies using inbred rat models of hypertension have identified BP quantitative trait loci (QTLs) on nearly every rat chromosome ${ }^{13-19}$ (http:// www.rgd.mcw.edu/). In our lab, we have resolved three of these QTLs and identified short genomic segments of 42.5, 177 and $804.6 \mathrm{~kb}$ containing genes coding for Rififylin, $11 \beta$-hydroxylase and Adamts16, respectively, as novel risk factors for hypertension. ${ }^{20-23}$
These studies were conducted using a hypertensive rat as one of the parental strains and a normotensive rat as the other parental strain.

Two of the most widely used rat models of hypertension are the Dahl Salt-sensitive (S) rat and the Spontaneously Hypertensive Rat (SHR). ${ }^{24}$ The S and SHR strains are both hypertensive models, but they exhibit differential development of hypertension. The BP of the $S$ rat, but not the SHR is exacerbated by a high-salt diet. ${ }^{25,26}$ In addition, SHR rats are resistant to the kidney damage that is seen in S rats. ${ }^{25-27}$ These phenotypic differences observed between S and SHR suggest that there are different genetic elements governing $\mathrm{BP}$ control in $\mathrm{S}$ and SHR rats. Previously, we identified two such genetic elements as BP QTLs on rat chromosome 9 (RNO9), in which SHR alleles on the S genetic background lower the $\mathrm{BP}$ of $\mathrm{S}$ rats on a $2 \% \mathrm{NaCl}$ diet. ${ }^{28}$ These two BP QTLs covered long genomic segments of 22.5 and $13.1 \mathrm{Mb}$ and contained 222 and 119 annotations, respectively. The purpose of the

${ }^{1}$ Physiological Genomics Laboratory, University of Toledo College of Medicine and Life Sciences, Toledo, OH, USA and ${ }^{2}$ Center for Diabetes and Endocrine Research, Department of Physiology and Pharmacology, University of Toledo College of Medicine and Life Sciences, Toledo, OH, USA

Correspondence: Dr B Joe, Physiological Genomics Laboratory, Department of Physiology and Pharmacology, University of Toledo College of Medicine, 3000 Arlington Avenue, Toledo, OH 43614-2598, USA.

E-mail: bina.joe@utoledo.edu

Received 22 February 2011; revised 19 May 2011; accepted 24 May 2011; published online 4 August 2011 
current study was to utilize the S.SHR congenic strains constructed in the previous study as tools to generate panels of congenic substrains and further map each of the BP QTLs to shorter genomic segments. Data are presented that support further resolution of two distinct BP QTLs to short genomic segments of 2.05 and $6.14 \mathrm{Mb}$. In addition, methodical characterization of the shorter of the two segments $(2.05 \mathrm{Mb})$ lending support to suggest that the expression of a novel gene, Tmeff 2 is linked to the development of hypertension in the $\mathrm{S}$ rat, is presented.

\section{METHODS}

\section{Animals}

All animal procedures and protocols used in this report were approved by the University of Toledo Health Science Campus Institutional Animal Care and Use Committee. The, Spontaneously Hypertensive Rat (SHR/Hsd), was originally obtained from Harlan Sprague-Dawley (Indianapolis, IN, USA) and maintained in our colony. The Dahl salt-sensitive rat (SS/jr) from our colony was used in all experiments and is referred to as $\mathrm{S}$. The panel of congenic substrains reported in this study was generated from S.SHR (9) x8A and S.SHR (9) x10A ${ }^{28}$ as previously described. ${ }^{21}$

\section{Genotyping}

PCR genotyping using microsatellite markers was done as previously described. ${ }^{19}$

\section{Tail-cuff BP measurement}

The experimental design for BP measurement by the tail-cuff method was as described previously. ${ }^{28}$ Briefly, at 30 days of age rats were weaned onto a low salt $(0.3 \% \mathrm{NaCl})$ Harlan Teklad 7034 diet. One or two congenic strains along with $\mathrm{S}$ rats were housed two to a cage such that two different strains were in each cage. At $40-42$ days of age the rats were switched to a $2 \% \mathrm{NaCl}$ diet (Harlan Teklad, TD 94217) and maintained on this diet for the duration of the experiment. During days $25-28$ on the $2 \% \mathrm{NaCl}$ diet, each rat had its systolic BP measured by two blinded operators. During BP measurements, rats were restrained and warmed to $28^{\circ} \mathrm{C}$. The operators' readings for each rat were averaged together and recorded as that animal's systolic BP. The following new congenic substrains were concomitantly raised and tested together for BP along with S rats: S.SHR(9)x8Ax6 and S.SHR(9)x8Ax12; S.SHR(9)x10Ax1 and S.SHR(9)x10Ax2; and S.SHR(9)x10Ax3 and S.SHR(9)x10Ax4.

\section{Telemetry}

$\mathrm{BP}$ was also collected using a telemetry system (Data Sciences International, St. Paul, MN, USA) as explained in detail previously. ${ }^{29}$ Briefly, 4 days after the BP measurements by the tail-cuff method, rats were surgically implanted with telemetry probes through their femoral arteries into the lower abdominal aortae. Rats were allowed to recover from surgery for 4 days before the transmitters were turned on for recording BP. All statistical analyses were as previously conducted. ${ }^{29}$

\section{Urinary protein excretion (UPE)}

UPE determination was done as previously described. ${ }^{26,30}$ Briefly, within 5 days following BP measurements, each rat was housed individually in a metabolic cage and its urine was collected over a 24-h period. The pyrogallol based QuanTtest Red Total Protein Assay from Quantimetrix (Redondo Beach, CA, USA) was used to determine protein concentrations of the urine samples. A VERSAmax microplate reader from Molecular Devices (Sunnyvale, CA, USA) was used to determine absorbance at $600 \mathrm{~nm}$. Protein concentrations were determined by reading against the absorbance of the QuanTtest human protein standards $\left(25-200 \mathrm{mg} \mathrm{dl}^{-1}\right)$. UPE data are presented as milligrams over a $24-\mathrm{h}$ period (mg per $24 \mathrm{~h})$.

\section{Echocardiography}

Left-ventricular function of $S(n=6)$ and congenic rats $(n=7)$ were evaluated by echocardiography as described previously. . $^{31,32}$

\section{Survival study}

S $(n=8)$ and S.SHR.(9)X8AX11A ( $n=8)$ rats were raised and administered 2\% $\mathrm{NaCl}$ containing diet as described under the BP measurements section. These rats were continued on the $2 \% \mathrm{NaCl}$ diet until their natural death.

\section{Gene sequencing}

DNA samples were obtained by tail biopsies as previously described ${ }^{19}$ from S.SHR(9)x8Ax11A, SHR and S. Primers were designed for the exons of Tmeff2 and LOC685674 using ExonPrimer (http://ihg.gsf.de/ihg/ExonPrimer.html). M13 primer sequences were added during the commercial synthesis of these custom exon primers by Integrated DNA Technologies (IDT, Coralville, IA, USA). PCR reactions were carried out using Platinum Taq High Fidelity DNA
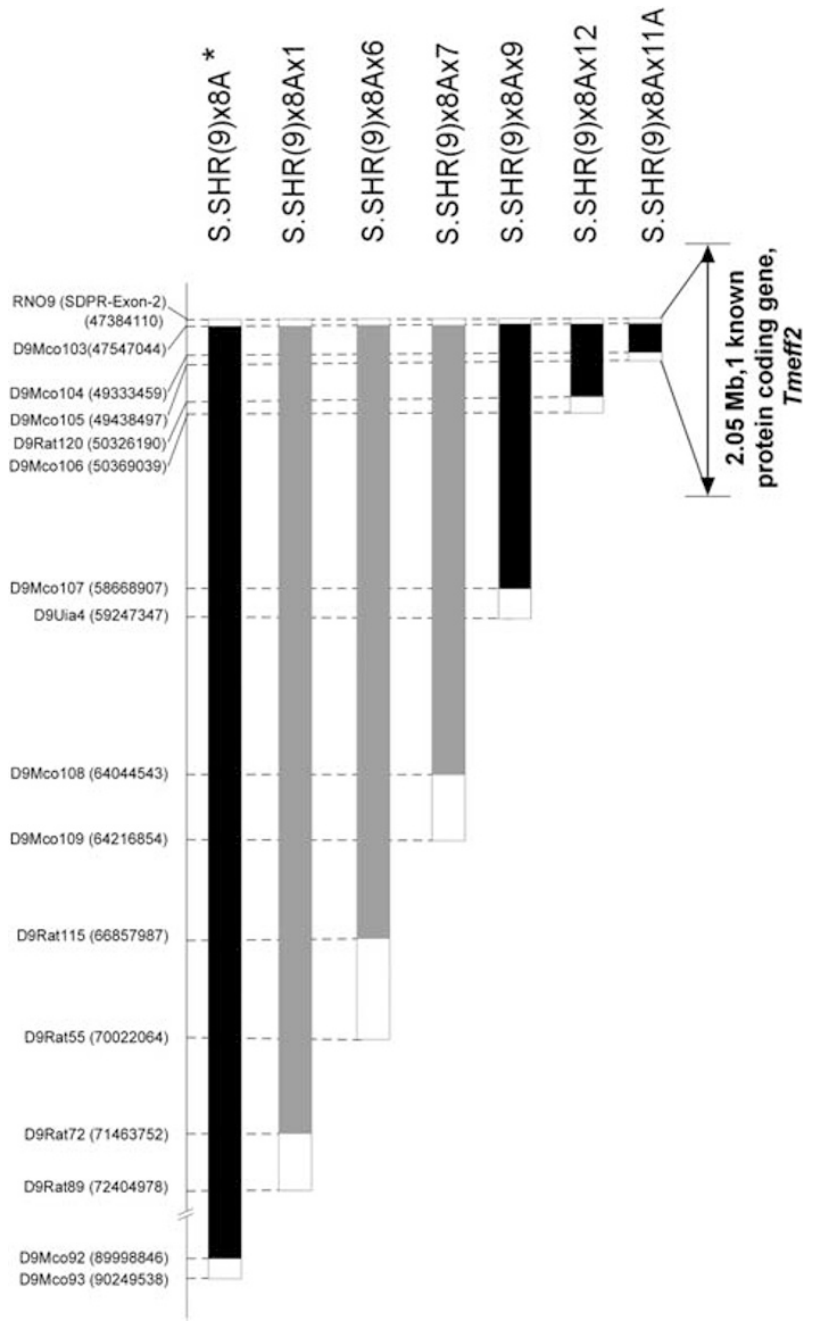

Figure 1 Schematic representation of the first set of newly developed congenic substrains. Congenic substrains were developed from S.SHR(9)x8A as described under Methods. The physical map of rat chromosome 9 is shown on the left with locations of markers in base pairs shown within parenthesis. Newly developed polymorphic microsatellite markers are given D9Mco numbers. Markers that begin with RNO9 are single-nucleotide polymorphic markers. RNO9 (SDPR-Exon 2) is a $\mathrm{C} / T$ single-nucleotide polymorphism between S and SHR. *The S.SHR(9)x8A congenic strain was previously reported and is shown here for reference. The solid bars represent SHR introgressed segments of the congenic strains. Open boxes flanking the solid bars are where recombinations occurred. The blood pressure (BP) data obtained from these congenic substrains are provided in Table 1. Black bars indicate congenic strains with BP significantly lower than that of the S. Gray bars indicate congenic strains with no BP effect. 
Table 1 Blood pressure effects of S.SHR(9) congenic substrains

\begin{tabular}{|c|c|c|c|c|c|c|c|c|}
\hline \multirow[b]{2}{*}{ Congenic substrain } & \multirow[b]{2}{*}{$S$} & \multicolumn{2}{|c|}{ Blood pressure $(\mathrm{mm} \mathrm{Hg})$} & \multirow[b]{2}{*}{$P$} & \multirow[b]{2}{*}{$S$} & \multicolumn{2}{|c|}{ Body Weight (g) } & \multirow[b]{2}{*}{$P$} \\
\hline & & Congenic & Effect & & & Congenic & Effect & \\
\hline S.SHR(9)x8Ax6 & $197[3.53]$ & 188 [3.67] & $-9[5.61]$ & 0.293 & 307 [4.93] & 302 [2.88] & $-5[5.74]$ & 0.689 \\
\hline S.SHR(9)x8Ax7 & $197[3.85]$ & $188[3.21]$ & $-9[5.01]$ & 0.068 & $312[2.80]$ & $307[2.84]$ & -5 [3.99] & 0.217 \\
\hline S.SHR(9)x8Ax9 & $214[5.26]$ & $181[3.91]$ & $-33[6.66]$ & $<0.001$ & $334[5.60]$ & $324[2.30]$ & $-10[6.05]$ & 0.132 \\
\hline S.SHR(9)x8Ax12 & $197[3.53]$ & $176[5.24]$ & $-21[5.87]$ & 0.003 & 307 [4.93] & $300[4.41]$ & $-7[6.01]$ & 0.547 \\
\hline S.SHR(9)x10Ax 1 & $200[4.22]$ & 185 [4.84] & $-15[6.22]$ & 0.039 & $353[6.24]$ & 339 [3.37] & $-14[6.86]$ & 0.095 \\
\hline S.SHR(9)x10Ax2 & $200[4.22]$ & $183[4.14]$ & $-17[6.13]$ & 0.014 & $353[6.24]$ & $347[4.08]$ & $-6[6.76]$ & 0.622 \\
\hline S.SHR(9)x10Ax3 & $213[5.14]$ & $208[5.78]$ & $-5[7.73]$ & 0.912 & $367[6.23]$ & $367[3.77]$ & $0[6.85]$ & 1.00 \\
\hline S.SHR(9)x $10 A \times 4$ & $213[5.14]$ & $203[6.11]$ & $-10[7.73]$ & 0.559 & $367[6.23]$ & $365[3.84]$ & $-2[6.85]$ & 0.995 \\
\hline
\end{tabular}

\begin{tabular}{|c|c|c|c|c|c|c|c|c|}
\hline & \multicolumn{3}{|c|}{ Heart Weight (g) } & \multirow[b]{2}{*}{$\mathrm{P}$} & \multirow[b]{2}{*}{$S$} & \multicolumn{2}{|c|}{ Relative Heart Weight } & \multirow[b]{2}{*}{$\mathrm{P}$} \\
\hline & $s$ & Congenic & Effect & & & Congenic & Effect & \\
\hline S.SHR(9)x8Ax 1 & $\mathrm{nr}$ & $\mathrm{nr}$ & - & - & $\mathrm{nr}$ & $\mathrm{nr}$ & - & - \\
\hline S.SHR(9)x8Ax7 & $1.27[0.020]$ & $1.23[0.014]$ & $-0.04[0.000]$ & 0.088 & $4.09[0.079]$ & $4.01[0.031]$ & $-0.08[0.085]$ & 0.348 \\
\hline S.SHR(9)×8Ax9 & $1.38[0.016]$ & $1.21[0.018]$ & $-0.17[0.024]$ & $<0.001$ & $4.16[0.065]$ & $3.75[0.063]$ & $-0.41[0.091]$ & $<0.001$ \\
\hline S.SHR(9)x8Ax11A & $1.68[0.058]$ & $1.40[0.038]$ & $-0.28[0.066]$ & $<0.001$ & $5.82[0.423]$ & $4.30[0.156]$ & $-1.52[0.391]$ & 0.001 \\
\hline S.SHR(9)x10Ax1 & $1.53[0.019]$ & $1.38[0.021]$ & $-0.15[0.031]$ & $<0.001$ & $4.36[0.073]$ & 4.09 [0.067] & $-0.27[0.100]$ & 0.024 \\
\hline S.SHR(9)x10Ax2 & $1.53[0.019]$ & $1.43[0.025]$ & $-0.10[0.031]$ & 0.004 & $4.36[0.073]$ & $4.13[0.070]$ & $-0.23[0.098]$ & 0.060 \\
\hline S.SHR(9)x10Ax3 & $1.55[0.019]$ & $1.52[0.013]$ & $-0.02[0.029]$ & 0.860 & $4.24[0.098]$ & $4.16[0.059]$ & $-0.08[0.113]$ & 0.893 \\
\hline S.SHR(9)x10Ax4 & $1.55[0.019]$ & $1.50[0.019]$ & $-0.04[0.029]$ & 0.393 & $4.24[0.098]$ & $4.12[0.072]$ & $-0.12[0.113]$ & 0.725 \\
\hline
\end{tabular}

Abbreviations: S, dahl salt-sensitive; SHR, spontaneously hypertensive rat.

Values are given as the mean with the standard error of the mean in brackets. The effect is defined as the Congenic mean minus the $\mathrm{S}$ mean. Effects were considered significant if $P$ value is $\leqslant 0.05$. Relative heart weight is defined as HW divided by BW times 1000. Body weight and heart weight were not recorded (nr) for S.SHR(9)x8Ax1.

Polymerase from Invitrogen (Carlsbad, CA, USA). The amplified products were resolved on 2\% agarose gels, purified using Qiaquick purification kits (Qiagen, Valencia, CA, USA), and sequenced commercially (MWG-http://www.operon.com). DNA sequences were analyzed using Sequencher (Genecodes Corporation, Version 4.9, Ann Arbor, MI, USA).

\section{RNA Isolation and RT-PCR analysis}

After the BP measurements by the tail-cuff method, heart, kidney and brain samples were obtained from S $(n=6)$ and congenic S.SHR(9)X8Ax11A $(n=6)$ rats. Total RNA was extracted using TRI reagent (Invitrogen). One microgram of total RNA was used to detect gene expression by Real-Time PCR (BioRad, Hercules, CA, USA) and expression levels relative to Gapdh were calculated by the $2^{-\Delta \Delta \mathrm{CT}}$ method. ${ }^{18}$

\section{5' RACE transcript analysis}

First-strand cDNA was synthesized from total RNA using a gene-specific primer (GSP1) and superscript ${ }^{\mathrm{TM}}$ II reverse (Invitrogen). The remaining RNA was degraded by Rnase $\mathrm{H}$ treatment after the completion of cDNA synthesis. cDNA was purified to remove unincorporated dNTP's, GSP1 and proteins using an SNAP column. A homopolymeric tail was then added to the $3^{\prime}$ end of the purified cDNA using TdT and dCTP. Then the tailed cDNA was used as template for subsequent PCR reaction using GSP2 that anneals to a site located within the cDNA molecule and a novel deoxyinosine containing anchor primer (provided by the manufacturer). Following amplification the PCR products were resolved by agarose gel electrophoresis, stained with ethidium bromide and the bands were visualized under UV-light.

\section{Immunoblot analysis}

Antibodies were from the following sources: goat polyclonal anti- Tmeff2 (N-15) (SC-47504), rabbit monoclonal anti-Gapdh (Cell Signaling, Danvers, MA, USA), bovine anti-rabbit IgG-HRP conjugate (SC-2370) and donkey antigoat IgG-HRP conjugate (Santa Cruz Biotechnology, Inc, Santa Cruz, CA, USA) (SC-2020). Samples were homogenized in ice-cold RIPA lysis buffer with protease inhibitor cocktail (Pierce, Rockford, IL, USA). Forty microgram of proteins were boiled with Laemmli loading buffer for 5 minutes at $95{ }^{\circ} \mathrm{C}$. Protein samples were resolved using 10\% NuPAGE Bis-Tris-gel (Invitrogen) at room temperature, and transferred on to PVDF membrane (Millipore, Temecula, CA, USA). The membrane was blocked with $3 \%$ BSA, incubated with primary and secondary antibodies before visualization by the chemiluminescence method (Pierce). Image J was used to quantify the protein expression level.

\section{Statistical analysis}

All BP statistical analyses were done using the SPSS software (SPSS, Chicago, IL, USA). Data were analyzed by the Student's $t$-test unless more than two strains were tested concomitantly, in which case, a one-way analysis of variance was used to assess statistical significance. Data are presented as the mean \pm standard error. A $P$-value of $\leqslant 0.05$ was used as a threshold for statistical significance. For BP and UPE experiments and subsequent heart weight and body weight measurements the number of rats in each group ranged from 10 to 20 . Survival analysis was done using the Kaplan-Meier analysis.

\section{RESULTS}

\section{Mapping a BP QTL within $2.05 \mathrm{Mb}$}

Using a congenic strain reported in our previous study, ${ }^{28}$ S.SHR (9)X8A, a new panel of congenic substrains of the $S$ rat containing 

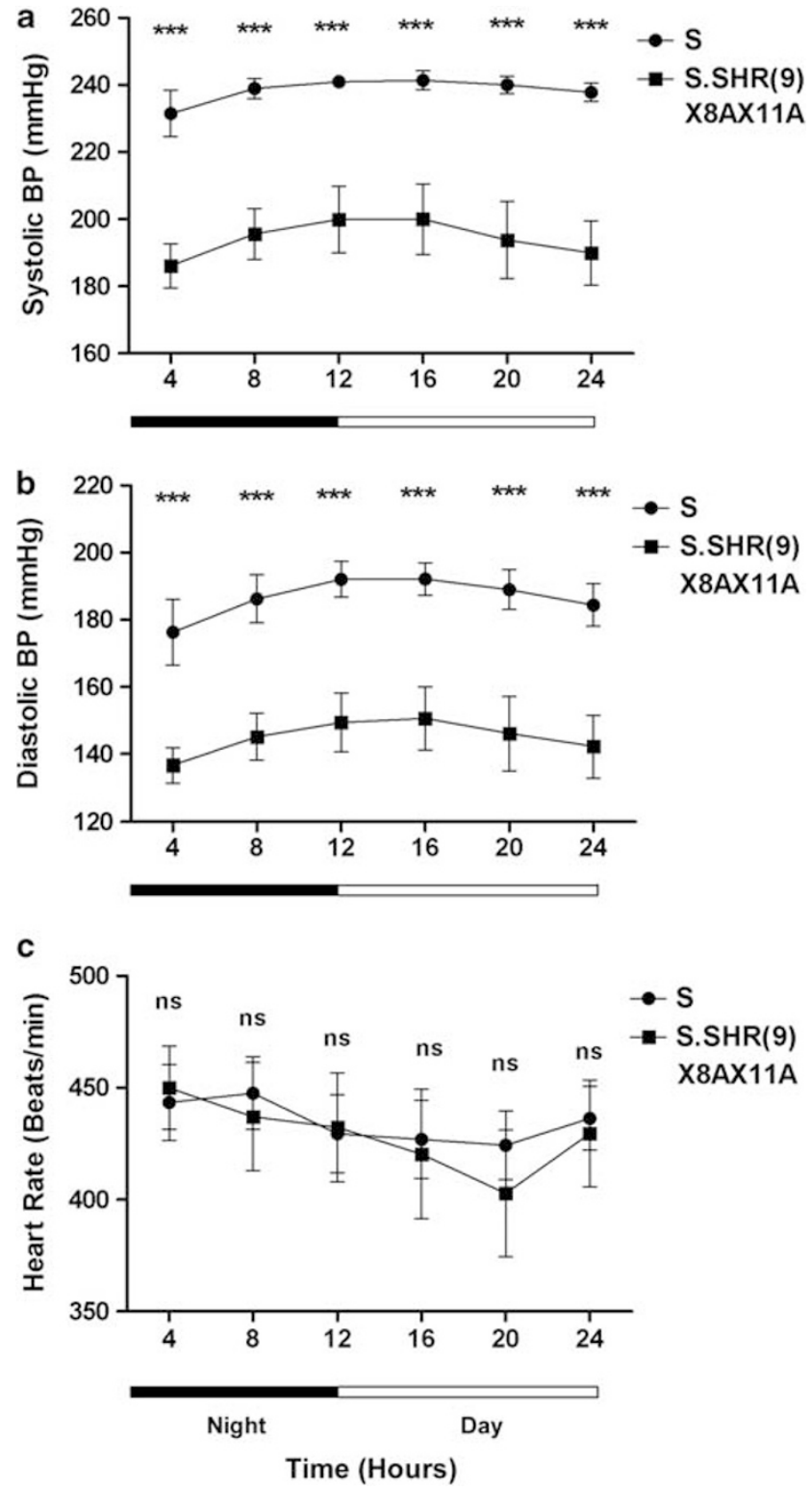

Figure 2 Radiotelemetry measures on (a) systolic BP, (b) diastolic BP (c) heart rate $S$ and S.SHR(9)x8Ax11A. Rats ( $n=6$ per group) were surgically implanted with $\mathrm{C} 40$ blood pressure radiotelemetry transmitters as described under Methods. Data points are 4-hour moving averages \pm s.e.m. $* * * P<0.001 ; \mathrm{ns}$, not significant.

introgressed segments of chromosome 9 from the SHR were generated. A schematic diagram of these new congenic substrains is shown in Figure 1. The BP of the congenic substrains shown in gray in Figure 1, S.SHR(9)x8Ax1, S.SHR(9)x8Ax7 and S.SHR(9)x8Ax6 were not significantly different from that of the $S$ (Table 1), whereas the BP of the three other strains shown in black in Figure 1, S.SHR(9)x8Ax9, S.SHR(9)x8Ax12 and S.SHR(9)x8Ax11A, were significantly lower than that of $S$ by 33, 21 and $56 \mathrm{~mm} \mathrm{Hg}$, respectively (Table 1). The three congenic substrains with BP lowering effects also had significantly lower relative heart weights compared with that of $S$ rats (Table 1). S.SHR(9)x8Ax11A was the congenic substrain with the shortest introgressed segment of $2054387 \mathrm{bp}$, which demonstrated a BP lowering effect (Figure 1). The BP study of this strain was repeated by the tail-cuff method. S.SHR(9)x8Ax11A had $39 \mathrm{~mm} \mathrm{Hg}$ lower BP com-
Table 2 Urinary protein excretion of S.SHR(9) congenic substrains

\begin{tabular}{|c|c|c|c|c|}
\hline \multirow[b]{2}{*}{ Congenic substrain } & \multicolumn{3}{|c|}{ Urinary protein excretion (mg per $24 \mathrm{~h}$ ) } & \multirow[b]{2}{*}{$\mathrm{P}$} \\
\hline & $S$ & Congenic & Effect & \\
\hline S.SHR(9)x8Ax1 & 78 [13.86] & 72 [5.442] & $-6[16.16]$ & 0.716 \\
\hline S.SHR(9)×8Ax6 & $102[6.81]$ & $86[5.72]$ & -16 [9.39] & 0.212 \\
\hline S.SHR(9)x8Ax7 & $127[10.32]$ & $87[8.11]$ & $-40[13.12]$ & 0.004 \\
\hline S.SHR(9)x8Ax9 & $94[7.54]$ & $69[4.55]$ & $-25[9.01]$ & 0.013 \\
\hline S.SHR(9)x8Ax11A & $253[20.37]$ & $212[14.66]$ & $-41[25.10]$ & 0.109 \\
\hline S.SHR(9)×8Ax12 & $102[6.81]$ & $72[8.09]$ & $-30[9.86]$ & 0.012 \\
\hline S.SHR(9)x10Ax1 & $160[6.78]$ & $135[7.39]$ & $-25[9.66]$ & 0.036 \\
\hline S.SHR(9)×10Ax2 & $160[6.78]$ & $140[5.78]$ & $-20[8.96]$ & 0.080 \\
\hline S.SHR(9) $\times 10 A \times 3$ & $\mathrm{nr}$ & $\mathrm{nr}$ & $\mathrm{nr}$ & $\mathrm{nr}$ \\
\hline S.SHR(9)×10Ax4 & $\mathrm{nr}$ & $\mathrm{nr}$ & $\mathrm{nr}$ & $\mathrm{nr}$ \\
\hline
\end{tabular}

Urinary protein excretion data are presented as milligrams over a $24 \mathrm{~h}$ period (mg per $24 \mathrm{~h}$ ). Values are given as the mean with the standard error of the mean in brackets. The effect is defined as the Congenic mean minus the $\mathrm{S}$ mean. Effects were considered significant if $P$ value is $P \leqslant 0.05$. Urinary protein excretion were not recorded (nr) for S.SHR(9)x10Ax3 and S.SHR(9)x10Ax4.

pared with $\mathrm{S}(P<0.001, n=20$ per group). Additional confirmation of the BP effect was sought by the radiotelemetry method (Figure 2). Both systolic and diastolic BP of S.SHR(9)x8Ax11A were significantly lower than that of the $S$ (Figure $2 \mathrm{a}$ and $\mathrm{b}$ ). Heart rates of S.SHR(9)x8Ax11A were, however, not different from that of the $S$ (Figure 2c). These data suggest that a major BP QTL is located within the $2.05 \mathrm{Mb}$ congenic segment of S.SHR(9)x8Ax11A.

The profiles of the panel of congenic substrains in Figure 1 compared with $\mathrm{S}$ were not the same for BP and UPE effects. Among the strains that lowered BP of the S, S.SHR(9)x8Ax9 and S.SHR(9)x8Ax12 had similar significant effects on UPE, reducing protein excretion by $25( \pm 9.01, P=0.013)$ and $30 \quad( \pm 9.86$, $P=0.012) \mathrm{mg}$ per $24 \mathrm{~h}$, respectively (Figure 1, Table 2). S.SHR(9)x8Ax11A did not affect UPE of the $S$ rat. The congenic substrain S.SHR(9)x8Ax7, which did not affect BP, reduced the UPE of the $S$ rat by $40( \pm 13.12, P=0.004) \mathrm{mg}$ per $24 \mathrm{~h}$. The only two strains that did not show either a BP effect or a UPE effect were S.SHR(9)x8Ax1 and S.SHR(9)x8Ax6 (Figure 1, Table 2).

\section{Fine-mapping a second closely linked BP QTL within 6.14 Mb} In our previous report, ${ }^{28}$ a second BP QTL distal to the region finemapped above was described. To fine-map this distal QTL, a second panel of congenic substrains were generated from the progenitor strain S.SHR(9)x10A shown in Figure 3. The congenic substrains S.SHR(9)x10Ax2 and S.SHR(9)x10x1 significantly lowered BP of the $S$ rat by $17( \pm 6.13, P=0.014)$ and $15 \mathrm{mmHg}( \pm 6.22, P=0.039$, Table 1). Two other substrains with shorter introgressed segments, S.SHR(9)x10Ax4 and S.SHR(9)x10Ax3 did not lower BP of the S rat (Table 2). The congenic substrain S.SHR(9)x10Ax1, which had the shortest introgressed segment and demonstrated a BP-lowering effect by the tail-cuff method, was further tested by the radiotelemetry approach. Systolic and diastolic BP of S.SHR(9)x10Ax1 was significantly lower than that of the $S$ (Figure $4 \mathrm{a}$ and $\mathrm{b}$ ). Further, heart rate of this strain was higher than that of the $S$ at all time points tested (Figure 4c). These data suggest that BP QTL2 is located within the congenic limits of S.SHR $(9) \times 10 A x 1$, which is $6.14 \mathrm{Mb}$. UPE of S.SHR(9)x10Ax1 was lower than that of the $S$ by $25 \pm 9.66 \mathrm{mg}$ per $24 \mathrm{~h}, P<0.036$ (Table 2), suggesting that unlike the BP QTL located within $2.05 \mathrm{Mb}$, this BP QTL is associated with UPE. For further characterization, we focused on the shorter of the two mapped QTLs, that is, the BP QTL within $2.05 \mathrm{Mb}$. 
Further physiological genomic characterization of the BP QTL within $2.05 \mathrm{Mb}$

To assess the impact of the BP lowering effect on heart function, $\mathrm{S}$ and congenic S.SHR(9)x8Ax11A animals were studied by echocardiography. The left-ventricular end-diastolic area, end-systolic area, posterior wall thickness and antero-septal wall thickness were significantly lower in the S.SHR(9)x8Ax11A compared with S (Table 3). These data corroborate the relative heart weight data of S.SHR(9)x8Ax11A (Table 1) and are reminiscent of cardiac hypertrophic effects driven by the observed differences in BP. Further, the Kaplan-Mier plot shown in Figure 5 suggests that the congenic strain S.SHR(9)x8Ax11A survived significantly longer than that of the S. The median survival of the $S$ was 108 days, while that of the congenic strain S.SHR(9)x8Ax11A was 146 days $(P<0.02$ by Kaplan-Meier analysis).

Sequencing of positional candidate genes

The $2.05 \mathrm{Mb}$ congenic interval of S.SHR(9)x8Ax11A contained one protein coding gene, Tmeff 2 - transmembrane protein with EGF-like and two follistatin-like domains. The other predicted annotations
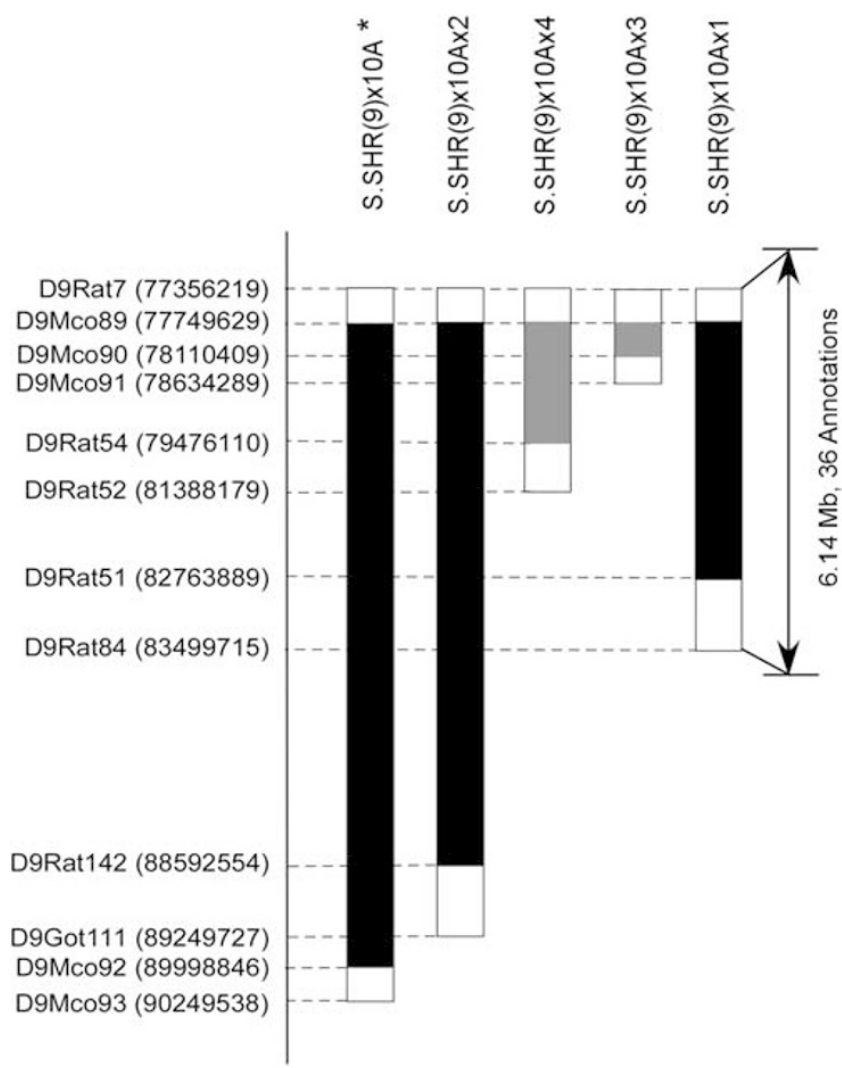

Figure 3 Schematic representation of the second set of newly developed congenic substrains. Congenic substrains were developed from S.SHR(9)x10A as described under Methods. The physical map of rat chromosome 9 is shown on the left with locations of microsatellite markers in base pairs shown within parenthesis. ${ }^{*}$ The S.SHR(9)x10A congenic strain was previously reported and is shown here for reference. The solid bars represent SHR introgressed segments of the congenic strains. Open boxes flanking the solid bars are where recombination's occurred. The blood pressure (BP) data obtained from these congenic substrains are provided in Table 1. Black bars indicate congenic strains with BP significantly lower than that of the S. Gray bars indicate congenic strains with no BP effect. included LOC685674, one exon of the gene Sdpr coding for serumdeprivation response protein, a pseudogene with no known protein product, two unknown predicted genes and five genes for small nuclear RNAs. The exons for Sdpr, Tmeff2, LOC685674 and a neighboring gene $(O f c 2 a)$ were sequenced, revealing no coding sequence variations. Note that the neighboring gene sequence of $O f c 2 a$ was completed before the detection of the SNP in the 3'-UTR of Sdpr, which delimits the top of the congenic segment of S.SHR(9)x8Ax11A (Figure 1). Detection of this SNP ruled out $O f c 2 a$ as a positional candidate within S.SHR(9)x8Ax11A.

\section{Characterization of the rat Tmeff 2 transcripts}

We next focused on differential expression of Tmeff 2 , the only known complete predicted protein-coding gene within the $2.05 \mathrm{Mb}$ critical
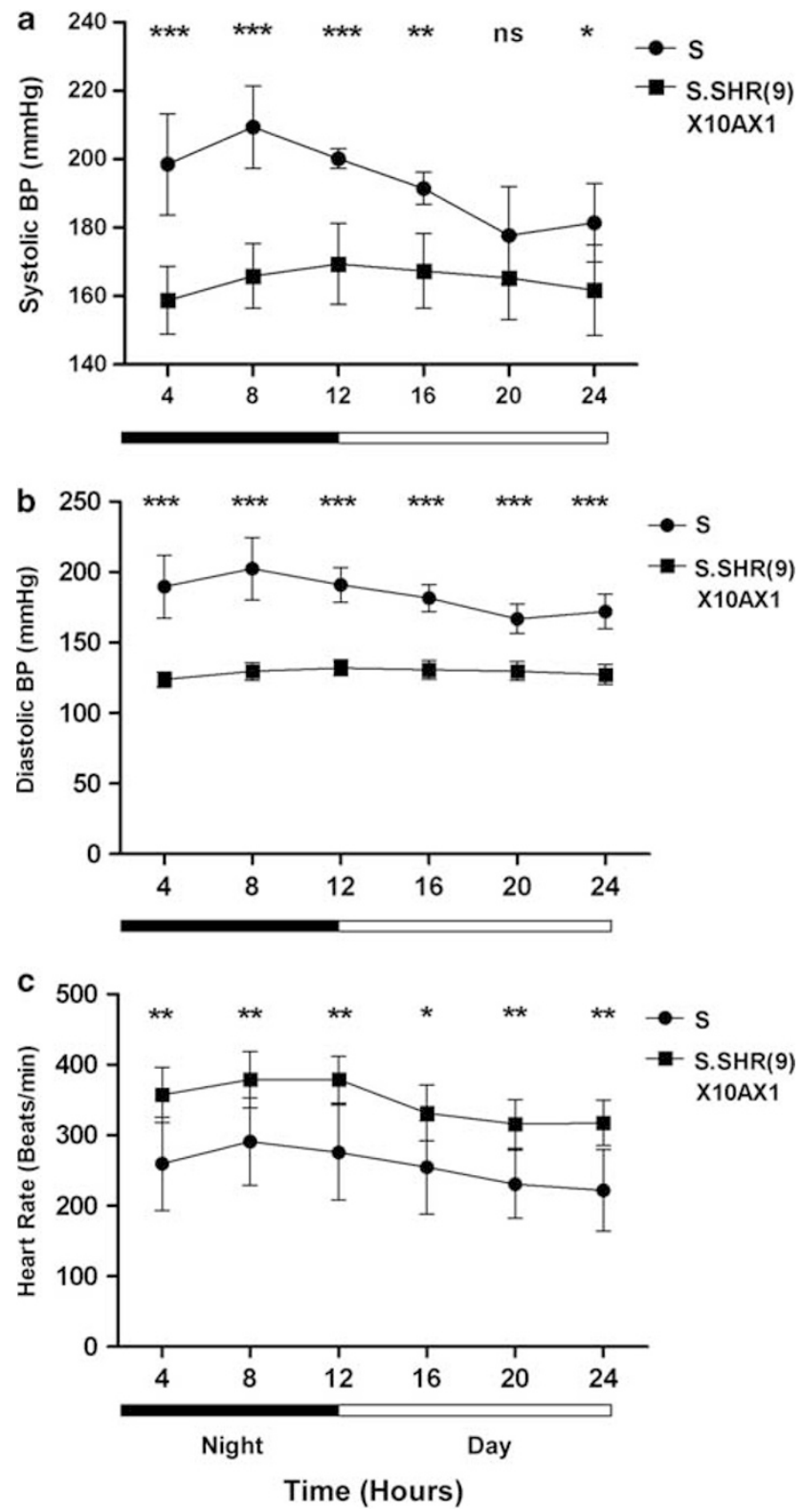

Figure 4 Radiotelemetry measures on (a) systolic BP, (b) diastolic BP (c) heart rate $S$ and S.SHR(9)x10Ax1. Rats ( $n=6$ per group) were surgically implanted with $\mathrm{C} 40$ blood pressure radiotelemetry transmitters as described under Methods. Data points are 4-hour moving averages \pm s.e.m. ${ }^{* * *} P<0.001 ;{ }^{*} P<0.01,{ }^{*} P<0.05, \mathrm{~ns}$, not significant. 
Table 3 Echocardiography of S and S.SHR(9)X8AX11A Congenic rats

\begin{tabular}{lllllr}
\hline Strain & $N$ & $E D A, \mathrm{~cm}^{2}$ & $E S A, \mathrm{~cm}^{2}$ & $P W T, \mathrm{~cm}$ & SWT, cm \\
\hline S & 6 & $0.62 \pm 0.11$ & $0.25 \pm 0.07$ & $0.29 \pm 0.03$ & $0.26 \pm 0.02$ \\
S.SHR(9)×8Ax11A & 7 & $0.88 \pm 0.06^{\dagger}$ & $0.44 \pm 0.05^{\dagger}$ & $0.24 \pm 0.01^{\dagger}$ & $0.20 \pm 0.01^{\dagger}$ \\
\hline
\end{tabular}

Abbreviations: EDA, end-diastolic area; ESA, end-systolic area; LV, left ventricular; N, Number of samples; PWT, posterior wall thickness; RWT, relative wall thickness; SWT, antero-septal wall thickness.

Data are presented as the mean \pm s.e.m. ${ }^{\dagger} P<0.05$ vs. S rats.

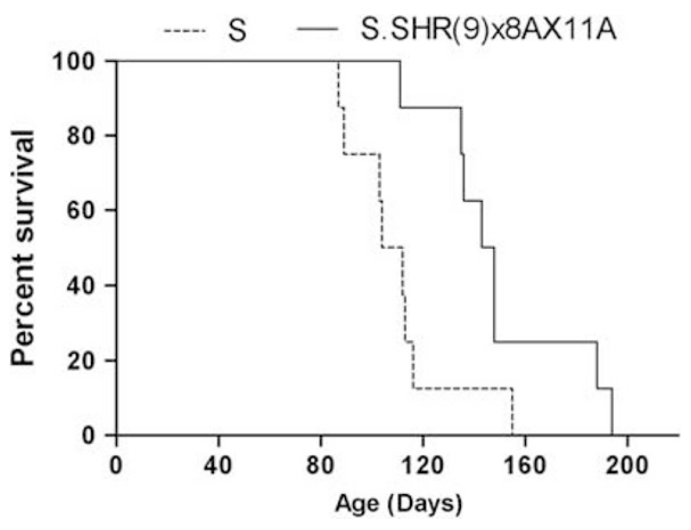

Figure 5 Comparison of survival times of $S$ vs. S.SHR(9)×8Ax11A. Rats ( $n=8$ per group) were studied for survival as described under Methods. ${ }^{*} P<0.02$ by the Kaplan-Meier analysis.

interval. The Ensembl database (www.ensembl.org) predicted three transcripts of Tmeff2 (Figure 6a). Primers were designed to amplify segments that are unique to each of these transcripts (Figure 6a). PCR reactions with cDNA isolated from the heart resulted in products for only two out of these three predicted transcripts (Figure 6b). Similar data were obtained from kidney samples also (data not shown). The $139 \mathrm{bp}$ and $118 \mathrm{bp}$ PCR products (Figure $6 \mathrm{~b}$ ) were confirmed to belong to Tmeff 2 by cDNA sequencing. To quantitate these transcripts, RNA samples from $S$ and $\operatorname{S.SHR}(9) \times 8 A x 11 A$ were assessed by real-time PCR. The mRNA levels of the full-length transcript ENSRNOT00000066231 were highly expressed in the brain, but were not differential between $S$ and S.SHR(9)x8Ax11A. However, both in the hearts and kidneys, the Tmeff2 transcript, ENSRNOT00000066231, was significantly higher in the S compared with S.SHR(9)x8Ax11A (Figure 6c). To ascertain the $5^{\prime}$-end prediction of ENSRNOT00000066231, a 5'-RACE experiment was conducted. Amplification and sequencing of the 585bp 5'-RACE PCR product shown in Figure $6 \mathrm{~d}$ confirmed that the start-site of this transcript was correct as predicted on the Ensembl website (www.ensembl.org). Further, the protein product of the Tmeff 2 transcript ENSRNOT00000066231 was significantly higher only in the hearts of S compared with S.SHR(9)x8Ax11A (Figures 6e and f).

\section{DISCUSSION}

Using congenic strains introgressing SHR alleles onto the S genetic background we previously identified two BP QTLs on RNO9. ${ }^{28}$ Mapping studies where differential congenic segments were used to determine the location of BP QTLs are increasingly recognized as not being ideal. ${ }^{13,33}$ Therefore, to further resolve the two BP QTLs, we developed two new iterations of congenic substrains for each of the BP QTLs and interpreted the location of the QTLs as the introgressed regions within the shortest congenic strains with BP effects. Accordingly, two distinct short genomic segments of 2.05 and $6.14 \mathrm{Mb}$ on
RNO9 were resolved as BP QTL containing congenic segments. The $6.14 \mathrm{Mb}$ segment contained 39 annotations and requires further genetic dissection. Therefore, the current study was further focused on the shorter of the two segments, that is, $2.05 \mathrm{Mb}$.

The single known and complete predicted protein-coding gene retained within the congenic segment was experimentally verified as a rat gene coding for at least two alternate transcripts of Tomoregulin-2, also known as a transmembrane glycoprotein with EGF-like and two Follistatin-like domains 2 (Tmeff2). Tmeff2 is predominantly expressed in the brain, but the levels of Tmeff 2 expression were not different between the brains of $\mathrm{S}$ and the congenic strain S.SHR(9)x8Ax11A, which has SHR alleles at Tmeff2. Tmeff2 was also expressed in other tissues relevant to BP control such as the kidney and heart. The mRNA of the longest transcript of Tmeff 2 was upregulated in hearts of the $S$ rat, which demonstrated increased cardiac hypertrophy compared with the congenic strain carrying SHR alleles of Tmeff2. The protein product of this long transcript of Tmeff 2 was also higher only in the heart samples of S compared with the congenic strain. These results suggest that decreasing the expression of Tmeff 2 protein in the $S$ rat by introgression of SHR alleles of a genomic segment containing Tmeff 2 is associated with lowering of BP of the S.

Tmeff 2 has additional aliases, TENB2, HPP1, TR, TPEF and CT120.2, and is a known epigenetic factor modulating cancer-related phenotypes. ${ }^{34-36}$ It is also recognized as a trophic factor for dopaminergic mesenchephalic neurons. ${ }^{37}$ Human TMEFF2 contributes to cell proliferation in an ADAM17-dependent autocrine fashion in cells expressing this protein. ${ }^{38}$ Perhaps related to this observation, a recent genome-wide association study indicates that intronic single-nucleotide polymorphisms of TMEFF2 are associated with body mass index in humans. ${ }^{39}$ However, beyond the demonstrated alterations in expression of the transcript of Tmeff 2 in tissues other than the brain, information regarding the function of Tmeff 2 is limited. TMEFF2 contains two follistatin-like domains that directly interact with TGF- $\beta$ and regulate related growth factor signaling; ${ }^{36}$ it is possible that the increased TMEFF2 levels may allow for increased interaction with TGF- $\beta$ leading to alterations in fibrosis. Lack of a proteinuria effect in the congenic strain, S.SHR(9)x8Ax11A, containing SHR alleles at Tmeff2 suggests that the functional effect of Tmeff 2 related to BP regulation may be elicited from tissues other than the kidney. Significant decreases in left-ventricular diastolic and systolic area were observed in the $S$ rats with higher levels of both mRNA and protein expression of Tmeff 2 compared with the congenic with SHR alleles of Tmeff2. Therefore, the heart could be further investigated as one of the potential sites from where the functional effects of Tmeff 2 contribute to BP regulation. The translational significance of our work is that it is similar to the observation that the left ventricles of patients from multiple cohorts with hypertrophic cardiomyopathy had a 2.05 fold increase in mRNA of TMEFF2 compared with healthy individuals (http://www.ncbi.nlm.nih.gov/geo/; Geo Accession Number: GSE1145). The causal genetic element responsible for the differential expression of Tmeff 2 remains to be explored in both rats and in 

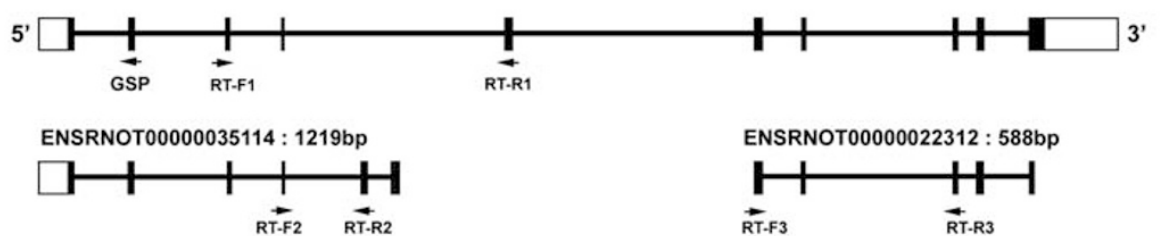

b
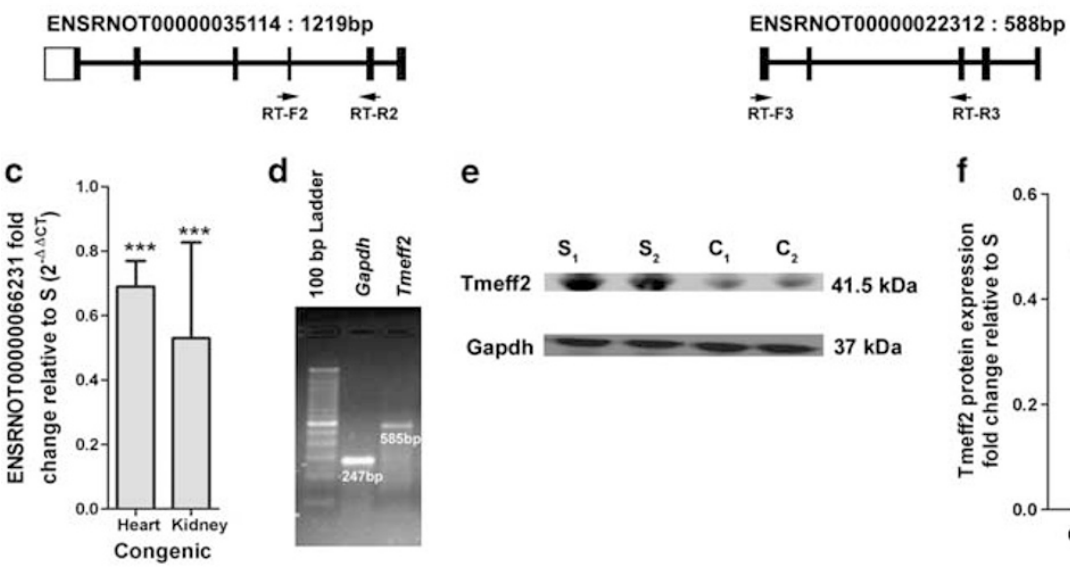

e

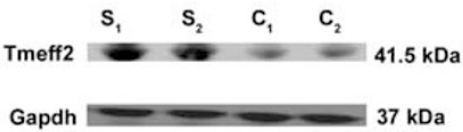

f

Figure 6 Transcript and protein expression analysis of Tmeff2 (Tomoregulin-2) in S and S.SHR(9)x8Ax11A. The schematics of the three predicted transcripts of Tmeff 2 from the Ensembl database (www.ensembl.org) are shown in Panel (a). Open white boxes are $5^{\prime}$ and $3^{\prime}$ untranslated regions. Thick vertical bars are exons. ENS numbers are transcript numbers assigned by the Ensembl database. GSP, location of gene-specific primer used for 5'RACE experiment; RTF1 and RT-R1, locations of primers designed to specifically amplify the full-length predicted transcript ENSRNOT00000066231. Expected product size $=118 \mathrm{bp}$ (b). RT-F2 and RT-R2, locations of primers designed to amplify the second predicted transcript ENSRNOT00000035114. No product amplified (b); RT-F3 and RT-R3, locations of primers designed to amplify the third predicted transcript ENSRNOT00000022312. Expected product size $=139 \mathrm{bp}$ (b). (c) is the real-time PCR data from S vs. S.SHR(9)x8Ax11A congenic substrain. mRNA obtained from heart and kidney samples were converted to cDNA and amplified using primers RT-F1 and RT-R1 shown in panel (a). Values are fold change in congenic relative to $\mathrm{S} \pm$ s.e.m. ${ }^{* * *} P<0.001$; (d) $5^{\prime}$ RACE analysis. The GSP primer represented in panel (a) was used in the 5' RACE experiment detailed under Methods. Expected product size of $585 \mathrm{bp}$ was obtained with the ENSRNOT00000066231 transcript of Tmeff2. Expected product size of $247 \mathrm{bp}$ was obtained with GAPDH used as the positive control. (e) Western blot analysis of heart samples from S and S.SHR(9)x8Ax11A. S1 and S2 are two different S rats. C1 and C2 are two different congenic rats. (f) Quantitation of the blots shown in panel (e); values are fold change in congenic relative to $S \pm$ s.e.m. ${ }^{* *} P<0.01$.

humans. These elements could be allelic variants within regulatory regions including non-coding RNA/s within the congenic interval. Nevertheless, the observation that differential expression of Tmeff 2 in the heart is linked to hypertension and cardiac hypertrophy is novel.

\section{ACKNOWLEDGEMENTS}

This work was funded by National Institutes of Health RO1 grants HL020176 and HL076709 to B Joe.

1 Franceschini N, Reiner AP, Heiss G. Recent Findings in the Genetics of Blood Pressure and Hypertension Traits. Am J Hypertens 2010; 24: 392-400.

2 Simino J, Shi G, Kume R, Schwander K, Province MA, Gu CC, Kardia S, Chakravarti A, Ehret G, Olshen RA, Turner ST, Ho LT, Zhu X, Jaquish C, Paltoo D, Cooper RS, Weder A, Curb JD, Boerwinkle E, Hunt SC, Rao DC. Five blood pressure Loci identified by an updated genome-wide linkage scan: meta-analysis of the family blood pressure program. Am J Hypertens 2011; 24: 347-354.

3 Adeyemo A, Gerry N, Chen G, Herbert A, Doumatey A, Huang H, Zhou J, Lashley K, Chen Y, Christman M, Rotimi C. A genome-wide association study of hypertension and blood pressure in African Americans. PLoS Genet 2009; 5: e1000564.

4 Org E, Eyheramendy S, Juhanson P, Gieger C, Lichtner P, Klopp N, Veldre G, Doring A Viigimaa M, Sober S, Tomberg K, Eckstein G, Kelgo P, Rebane T, Shaw-Hawkins S, Howard P, Onipinla A, Dobson RJ, Newhouse SJ, Brown M, Dominiczak A, Connell J, Samani N, Farrall M, Caulfield MJ, Munroe PB, Illig T, Wichmann HE, Meitinger T, Laan M. Genome-wide scan identifies $\mathrm{CDH} 13$ as a novel susceptibility locus contributing to blood pressure determination in two European populations. Hum Mol Genet 2009; 18: 2288-2296.

5 Sober S, Org E, Kepp K, Juhanson P, Eyheramendy S, Gieger C, Lichtner P, Klopp N, Veldre G, Viigimaa M, Doring A, Putku M, Kelgo P, Shaw-Hawkins S, Howard P, Onipinla A, Dobson RJ, Newhouse SJ, Brown M, Dominiczak A, Connell J, Samani N, Farrall M, Caulfield MJ, Munroe PB, Illig T, Wichmann HE, Meitinger T, Laan M. Targeting 160 candidate genes for blood pressure regulation with a genome-wide genotyping array. PLoS One 2009; 4: e6034.
6 Torkamani A, Topol EJ, Schork NJ. Pathway analysis of seven common diseases assessed by genome-wide association. Genomics 2008; 92: 265-272.

7 Browning BL, Browning SR. Haplotypic analysis of Wellcome Trust Case Control Consortium data. Hum Genet 2008; 123: 273-280.

8 Hiura Y, Tabara Y, Kokubo Y, Okamura T, Miki T, Tomoike H, Iwai N. A genome-wide association study of hypertension-related phenotypes in a Japanese population. Circ $J$ 2010; 74: 2353-2359.

9 Ehret GB. Genome-wide association studies: contribution of genomics to understanding blood pressure and essential hypertension. Curr Hypertens Rep 2010; 12: 17-25.

10 Hong KW, Jin HS, Lim JE, Kim S, Go MJ, Oh B. Recapitulation of two genomewide association studies on blood pressure and essential hypertension in the Korean population. J Hum Genet 2010; 55: 336-341.

11 Hong KW, Jin HS, Lim JE, Cho YS, Go MJ, Jung J, Lee JE, Choi J, Shin C, Hwang SY, Lee SH, Park HK, Oh B. Non-synonymous single-nucleotide polymorphisms associated with blood pressure and hypertension. J Hum Hypertens 2010; 24: 763-774.

12 Altshuler D, Daly MJ, Lander ES. Genetic mapping in human disease. Science 2008; 322: 881-888.

13 Gopalakrishnan K, Saikumar J, Peters CG, Kumarasamy S, Farms P, Yerga-Woolwine S, Toland EJ, Schnackel W, Giovannucci DR, Joe B. Defining a rat blood pressure quantitative trait locus to $a<81.8 \mathrm{~kb}$ congenic segment: comprehensive sequencing and renal transcriptome analysis. Physiol Genomics 2010; 42A: 153-161.

14 Garrett MR, Joe B, Dene H, Rapp JP. Identification of blood pressure quantitative trait loci that differentiate two hypertensive strains. J Hypertens 2002; 20: 2399-2406.

15 Garrett MR, Joe B. Genetic analysis of inherited hypertension in the rat. In: Dominiczak A, Connell J (eds). Genetics of Hypertension. Elsevier Science: Amsterdam, The Netherlands, 2006, pp 177-200.

16 Cowley Jr AW. The genetic dissection of essential hypertension. Nat Rev Genet 2006; 7: 829-840

17 Joe B, Garrett MR. Substitution mapping: using congenic strains to detect genes controlling blood pressure. In: Raizada MK, Paton JFR, Kasparov S, Katovich MJ (eds). Cardiovascular Genomics: Gene Mining for Pharmacogenomics and Gene Therapy. Humana Press Inc.: NJ, 2005, pp 41-58.

18 Saad Y, Garrett MR, Manickavasagam E, Yerga-Woolwine S, Farms P, Radecki T, Joe B. Fine-mapping and comprehensive transcript analysis reveals nonsynonymous variants within a novel $1.17 \mathrm{Mb}$ blood pressure QTL region on rat chromosome 10. Genomics 2007; 89: 343-353. 
19 Saad Y, Yerga-Woolwine S, Saikumar J, Farms P, Manickavasagam E, Toland EJ, Joe B. Congenic interval mapping of RNO10 reveals a complex cluster of closely-linked genetic determinants of blood pressure. Hypertension 2007; 50: 891-898.

20 Garrett MR, Rapp JP. Defining the blood pressure QTL on chromosome 7 in Dahl rats by a 177-kb congenic segment containing Cyp11b1. Mamm Genome 2003; 14: 268-273.

21 Joe B, Saad Y, Lee NH, Frank BC, Achinike OH, Luu TV, Gopalakrishnan K, Toland EJ, Farms P, Yerga-Woolwine S, Manickavasagam E, Rapp JP, Garrett MR, Coe D, Apte SS, Rankinen T, Perusse L, Ehret GB, Ganesh SK, Cooper RS, O'Connor A, Rice T, Weder AB, Chakravarti A, Rao DC, Bouchard C. Positional identification of variants of Adamts16 linked to inherited hypertension. Hum Mol Genet 2009; 18 : 2825-2838.

22 Cicila GT, Rapp JP, Wang JM, St Lezin E, Ng SC, Kurtz TW. Linkage of 11 betahydroxylase mutations with altered steroid biosynthesis and blood pressure in the Dahl rat. Nat Genet 1993; 3: 346-353.

23 Gopalakrishnan K, Morgan EE, Yerga-Woolwine S, Farms P, Kumarasamy S, Kalinoski A, Liu X, Wu J, Liu L, Joe B. Augmented Rififylin Is a Risk Factor Linked to Aberrant Cardiomyocyte Function, Short-QT Interval and Hypertension. Hypertension 2011; 57: 764-771.

24 Rapp JP. Genetic analysis of inherited hypertension in the rat. Physiol Rev 2000; 80: $135-172$.

25 Garrett MR, Saad Y, Dene H, Rapp JP. Blood pressure QTL that differentiate Dahl saltsensitive and spontaneously hypertensive rats. Physiol Genomics 2000; 3: 33-38.

26 Garrett MR, Gunning WT, Radecki T, Richard A. Dissection of a genetic locus influencing renal function in the rat and its concordance with kidney disease loci on human chromosome 1q21. Physiol Genomics 2007; 30: 322-334.

27 Garrett MR, Dene H, Rapp JP. Time-course genetic analysis of albuminuria in Dahl saltsensitive rats on low-salt diet. J Am Soc Nephrol 2003; 14: 1175-1187.

28 Toland EJ, Yerga-Woolwine S, Farms P, Cicila GT, Saad Y, Joe B. Blood pressure and proteinuria effects of multiple quantitative trait loci on rat chromosome 9 that differentiate the spontaneously hypertensive rat from the Dahl salt-sensitive rat. J Hypertens 2008; 26: 2134-2141.

29 Joe B, Garrett MR, Dene H, Rapp JP. Substitution mapping of a blood pressure quantitative trait locus to a $2.73 \mathrm{Mb}$ region on rat chromosome 1 . J Hypertens 2003; 21: 2077-2084.
30 Garrett MR, Joe B, Yerga-Woolwine S. Genetic linkage of urinary albumin excretion in Dahl salt-sensitive rats: influence of dietary salt and confirmation using congenic strains. Physiol Genomics 2006; 25: 39-49.

31 Cicila GT, Morgan EE, Lee SJ, Farms P, Yerga-Woolwine S, Toland EJ, Ramdath RS, Gopalakrishnan K, Bohman K, Nestor-Kalinoski AL, Khuder SA, Joe B. Epistatic genetic determinants of blood pressure and mortality in a salt-sensitive hypertension model. Hypertension 2009; 53: 725-732.

32 Morgan EE, Faulx MD, McElfresh TA, Kung TA, Zawaneh MS, Stanley WC, Chandler MP, Hoit BD. Validation of echocardiographic methods for assessing left ventricular dysfunction in rats with myocardial infarction. Am J Physiol Heart Circ Physiol 2004; 287: H2049-H2053.

33 Shao H, Sinasac DS, Burrage LC, Hodges CA, Supelak PJ, Palmert MR, Moreno C, Cowley Jr AW, Jacob HJ, Nadeau JH. Analyzing complex traits with congenic strains. Mamm Genome 2010; 21: 276-286.

34 Gery S, Sawyers CL, Agus DB, Said JW, Koeffler HP. TMEFF2 is an androgen-regulated gene exhibiting antiproliferative effects in prostate cancer cells. Oncogene 2002; 21: 4739-4746.

35 Elahi A, Zhang L, Yeatman TJ, Gery S, Sebti S, Shibata D. HPP1-mediated tumor suppression requires activation of STAT1 pathways. Int J Cancer 2008; 122: 1567-1572.

36 Suzuki M, Shigematsu H, Shames DS, Sunaga N, Takahashi T, Shivapurkar N, lizasa T, Frenkel EP, Minna JD, Fujisawa T, Gazdar AF. DNA methylation-associated inactivation of TGFbeta-related genes DRM/Gremlin, RUNX3, and HPP1 in human cancers. Br J Cancer 2005; 93: 1029-1037.

37 Horie M, Mitsumoto Y, Kyushiki H, Kanemoto N, Watanabe A, Taniguchi Y, Nishino N, Okamoto T, Kondo M, Mori T, Noguchi K, Nakamura Y, Takahashi E, Tanigami A. Identification and characterization of TMEFF2, a novel survival factor for hippocampal and mesencephalic neurons. Genomics 2000; 67: 146-152.

38 Ali N, Knauper V. Phorbol ester-induced shedding of the prostate cancer marker transmembrane protein with epidermal growth factor and two follistatin motifs 2 is mediated by the disintegrin and metalloproteinase-17. J Biol Chem 2007; 282: 37378-37388.

39 Talbert M, Wing M, Allred N, Ziegler J, Langefeld C, Wagenknect L, Taylor K, Norris J, Haritunians T, Bryer-Ash M, Bowden D. Genome-wide association study of body mass index and follow-up candidate genotyping in African Americans: The IRAS Family Study. The 59th Annual Meeting of the American Society of Human Genetics, http://www.ashg.org/2009meeting/abstracts/fulltext/f20291.htm (abstract). 